\title{
Nanosizing of Model Drug: Characterization and Evaluation of Nanosized Model Drug Diffusion through Biomembrane
}

\section{Sushant Kumar ${ }^{1 *}$, Kamla Pathak ${ }^{1}$, NV Satheesh Madhav², Anurag Verma $^{3}$ and Swarnima Pandey ${ }^{4}$}

${ }^{1}$ Faculty of Pharmacy, Uttar Pradesh University of Medical Sciences, Saifai, Etawah, $U P$, India

${ }^{2}$ Faculty of Pharmacy, DIT University, Dehradun, Uttarakhand, India

${ }^{3}$ Faculty of Pharmacy, Teerthankar Mahaveer University, Moradabad, UP, India

${ }^{4}$ Goel Institute of Pharmacy \& Science, Dr. APJAKT University, Lucknow, India

*Corresponding Author: Sushant Kumar, Faculty of Pharmacy, Uttar Pradesh

University of Medical Sciences, Saifai, Etawah, U.P., India.
Received: April 28, 2021

Published: June 03, 2021

(C) All rights are reserved by Sushant Kumar., et al.

\section{Abstract}

Background: As the lamotrigine is poorly water soluble drug. The dug was characterized for different physico-chemical properties and nanosized by solvent evaporation sonication technique.

Aims and Objectives: This research was aimed to reduce the model particle size and evaluate the diffusion study.

Methods: In the research work the bath sonication was done at 250 hertz frequency at room temperature. During sonication $25 \mathrm{ml}$ of purified water as added slowly drop by drop till turbidity was observed. The obtained residue was then centrifuged for 30 minutes at $4000 \mathrm{rpm}$ for complete recovery of nanosized drug.

Results: Lamotrigine was found to be very soluble in methanol, soluble in phosphate buffer pH7.4 solution. It was found to be sparingly soluble in $0.1 \mathrm{MHCl}$, phosphate buffer, $\mathrm{pH} 5.8$ solutions. The nanosizing of drug particle was confirmed when the number of sonication cycles was increased up to 15 cycles as optimized, the \%transmittance was found to increase proportionally.

Conclusion: This diffusion was in significant amount and confirmed that the nanosized drug easily pass through the biomembrane and can be targeted to desired sites in significant amount.

Keywords: Lamotrigine; Nanosizing; SEM Testing; Diffusion; Solubility

\section{Introduction}

Nanotechnology has arisen as territory for examination of the fresher methodologies [1]. There are various novel properties of nanoparticles, for example, its nanoparticles size, custom-made surface, more noteworthy dissolvability, and its multifunctionality which shows its viability to interface with cell capacities recently and in this manner the conveyed nanoparticles can go into the cerebrum by intersection the BBB (Garcia., et al. 2005). Different medication atoms have been effectively and adequately conveyed utilizing nanoparticles as the transporter framework like for example dalargin, kytopherin, tubocurarine, doxorubicin. Polybutylcynaoacrylate nanoparticles covered with polysorbate 
80 were utilized for delivery of peptide utilized candidate. Various drugs have been tested by utilizing the equivalent nanoparticulate delivery systems. These nanoparticles move effectively and deliver the loaded drug straightforwardly to the brain microenvironment and afterward eventually biodegraded by the endocytotic technique.

\section{Advantages of nanoparticle}

There are several advantages of nanoparticle due to which they are preferred as nano drug delivery systems-

- The surface of nanoparticle can be modified for altering biodistribution of drugs for achieving subsequent clearance of drugs so that maximum therapeutic efficiency can be achieved with minimum side effects of drug [2].

- It is possible to modify the controlled release and particle degradation characteristics of nanoparticles systems by altering the choice of matrix systems.

- Drug loading is greater and drugs can be incorporated and loaded into the systems without any chemical reaction.

- The site specific drug targeting is possible by attaching targeting ligands or by using magnetic guidance

- Biodegradable liposomes and polymer based nanoparticles do not accumulate in body and so is risk free

- Nanoparticles can be administrated by various routes of administration such as oral, nasal, intraocular, parenteral routes etc. [3].

\section{Limitations of nanoparticles}

- Due to smaller particle size and greater surface area, nanoparticles are very reactive in cellular environment.

- Drug loading is limited and burst release problems are encountered due to smaller particle size. These problems have to be sorted out before formulation of nanoparticles.

\section{Nanosizing and it's applications}

The larger particle size before nanosizing in micrometer size range can be easily converted into nanoparticles size range in nanometer. Thus the nano size range also can improve the drug solubility as well as entrapment efficacy of the model drug. The nanosized drug can be significantly diffused through the egg biomembrane. This diffusion in significant amount and confirmed that the nanosized drug can easily pass through the biomembrane and can be targeted to desired sites in significant amount [3].

\section{Material and Methods}

\section{Materials}

The model drug lamotrigine was obtained as a gift sample from Affy pharma private limited, Baddi. All other chemicals used were of analytical grade.

\section{Physico-chemical characterization of model drug} Preformulation studies of lamotrigine

The solubility study of lamotrigine was performed in different solvents like purified water, methanol, phosphate buffer pH7.4, $0.1 \mathrm{M} \mathrm{HCl}$, phosphate buffer $\mathrm{pH} 5.8$. An excess quantity of lamotrigine was added gradually in $10 \mathrm{ml}$ of each solvent system in the beaker then placed on orbital shaker with the $100 \mathrm{rpm}$ rotation at $37^{\circ} \mathrm{C}$ for 24 hours. After shaking the dispersion was centrifuged for 10 minutes at $4000 \mathrm{rpm}$. Then the solution was filtered properly and the obtained filtrate was analyzed with UV spectrophotometer at $\lambda \max$ of $307 \mathrm{~nm}$. Result was interpreted and reported [4].

Preparation of calibration curve of lamotrigine in different solvent medium

Preparation of calibration curve of lamotrigine in methanol

For preparation of calibration curve of lamotrigine, $100 \mathrm{mg}$ of drug lamotrigine was weighed properly and was dissolved in 100 $\mathrm{ml}$ of methanol and labeled as $1 \mathrm{mg} / \mathrm{ml}$ stock solution. Suitable dilutions were made from the stock solution. The stock solution was used to prepare $5 \mu \mathrm{g} / \mathrm{ml}, 10 \mu \mathrm{g} / \mathrm{ml}, 15 \mu \mathrm{g} / \mathrm{ml}, 20 \mu \mathrm{g} / \mathrm{ml}$ and $30 \mu \mathrm{g} /$ $\mathrm{ml}$ using of methanol in $10 \mathrm{ml}$ volumetric flasks. The absorption maxima was determined from highest concentration of phenytoin. The absorbance was analyzed at $307 \mathrm{~nm} \lambda \max$ for different dilutions of drug [5].

Preparation of Calibration curve of lamotrigine in Phosphate buffer pH 7.4

In the same way the calibration curve in Phosphate buffer $\mathrm{pH}$ 7.4 was prepared. For this first $100 \mathrm{mg}$ of lamotrigine was taken accurately and dissolved in $100 \mathrm{ml}$ of methanol and labeled as $1 \mathrm{mg} /$ $\mathrm{ml}$ stock solution. The suitable dilutions were made to prepare 5 $\mu \mathrm{g} / \mathrm{ml}, 10 \mu \mathrm{g} / \mathrm{ml}, 15 \mu \mathrm{g} / \mathrm{ml}, 20 \mu \mathrm{g} / \mathrm{ml}$ and $30 \mu \mathrm{g} / \mathrm{ml}$ with the help of methanol in $10 \mathrm{ml}$ volumetric flasks. The absorbance was analyzed for different dilutions of drug at absorption maxima $307 \mathrm{~nm}$ [6]. 
Nanosizing of lamotrigine by modified solvent evaporation method

$500 \mathrm{mg}$ of Lamotrigine was taken and mixed with dextrose 50 $\mathrm{mg}$ in mortar in mortar and pestle and the $25 \mathrm{ml}$ of methanol was added to mixture. The clear solution mixture transferred in $50 \mathrm{ml}$ beaker and sonicated for 5 cycles (One cycle was for three minutes). This mixture was bath sonicated for optimized sonication cycle for 15 cycles (One cycle was for three minutes) continuously. The bath sonication was done at 250 hertz frequency at room temperature. During sonication $25 \mathrm{ml}$ of purified water as added slowly drop by drop till turbidity was observed. The obtained residue was then centrifuged for 30 minutes at $4000 \mathrm{rpm}$ for complete recovery of nanosized drug. During the optimization process at each cycle percentage transmittance was noted in order to know the minimum cycles required for nanosizing of the drug. After the completion of 15 cycles sonication and centrifugation the nanoparticles were collected and supernatant liquid was discarded. The collected nanoparticles was washed with water, properly dried and packed in air tight container $[2,7]$.

\section{Physical characterization of nanosized model drug}

The recovered nanoparticles were observed for physical appearance, permeability through egg membrane. Further the nanoparticles were stored in air tight container for further use. Then its permeability study was conducted for confirmation of its suitability for passage through the biomembrane in nano range [2].

\section{SEM characterization}

The nanosized drug was also characterized by SEM (scanning electron microscopy) testing $[6,7]$.

\section{Zeta particle size determination}

The nanosized drug was also characterized by Zeta sizer. The procedure of nanosizing has been summarized in flowchart 1 . The study was performed and reported [7].

\section{In-vitro permeability study through the egg membrane}

$5 \mathrm{mg} / \mathrm{ml}$ of nanosized lamotrigine solution was prepared in phosphate buffer $\mathrm{pH} 7.4$ and $4 \mathrm{ml}$ was taken in donor compartment and the donor compartment tip was tied with the isolated egg membrane. The phosphate buffer solution with pH 7.4 was added to the receptor compartment in diffusion assembly. The donor with

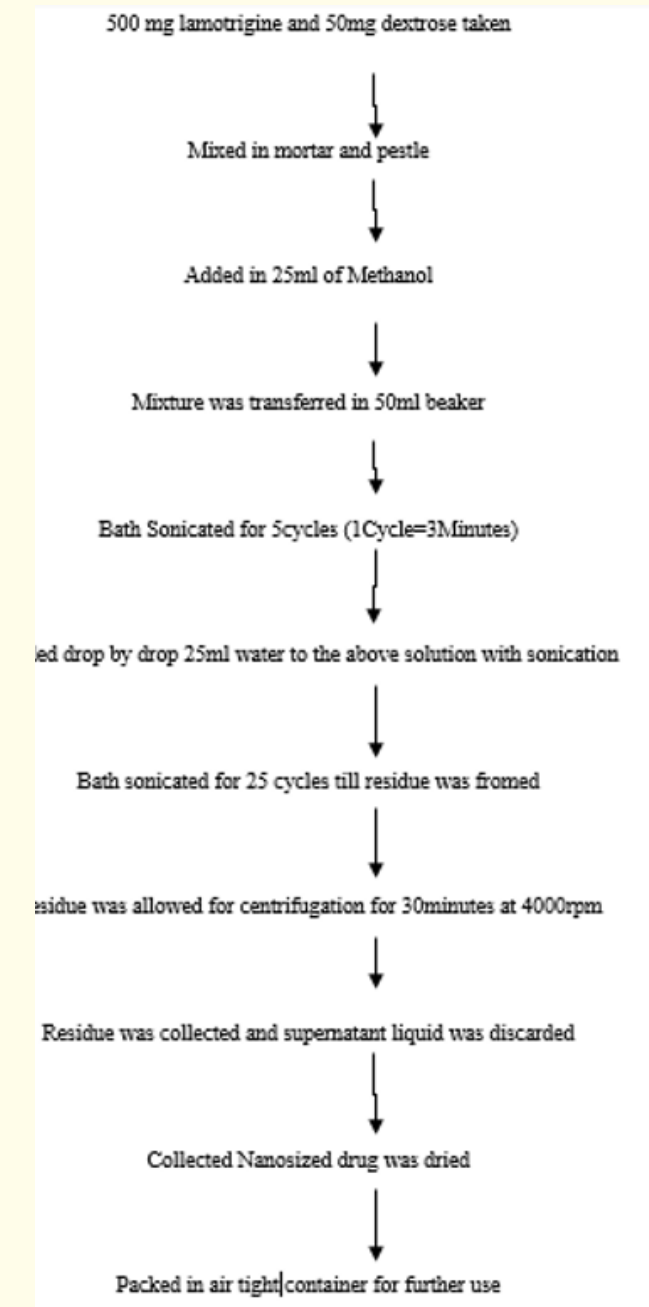

Flowchart 1: Nanosizing of Lamotrigine.

drug solution was fitted in receptor compartment for permeation of drug study. At specific time interval the receptor compartment sample was completely replaced with the fresh buffer solution. The sampling was done at 1, 2, 4, 6, 8, 10, 24, 36 and 48 hours and assayed. The graph was plotted between the time of permeation study and concentration in cumulative way of drug permeated at specific time interval. This procedure was done in triplicate. The In-vitro permeation study performed in assembly which has been shown in figure 1 . The study was performed and reported [1,8-10]. 


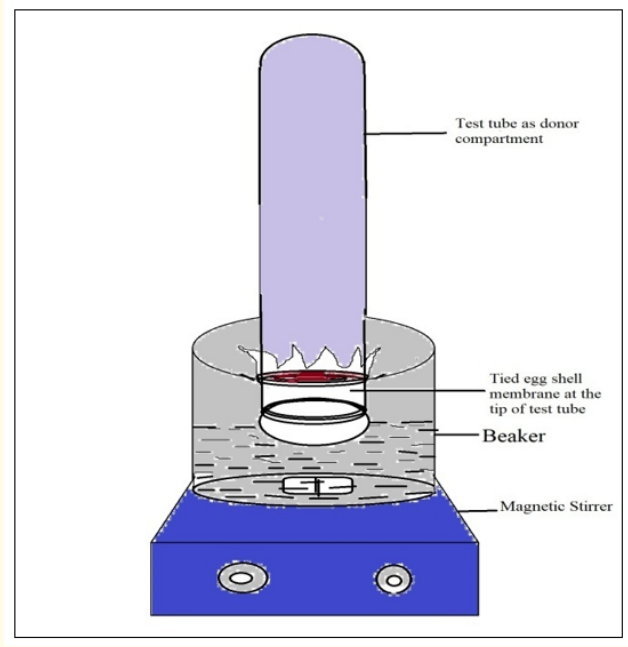

Figure 1: Assembly for In-vitro permeation study across egg membrane.

\section{Results and Discussion}

Physico-chemical characterization of model drug Preformulation studies of lamotrigine

\section{Solubility}

Lamotrigine was found to be very soluble in methanol, soluble in phosphate buffer pH7.4 solution. It was found to be sparingly soluble in $0.1 \mathrm{MHCl}$, phosphate buffer, pH 5.8 solutions [6]. It was also sparingly soluble in purified water (Figure 2). The solubility profile of the drug is summarised in table 1.

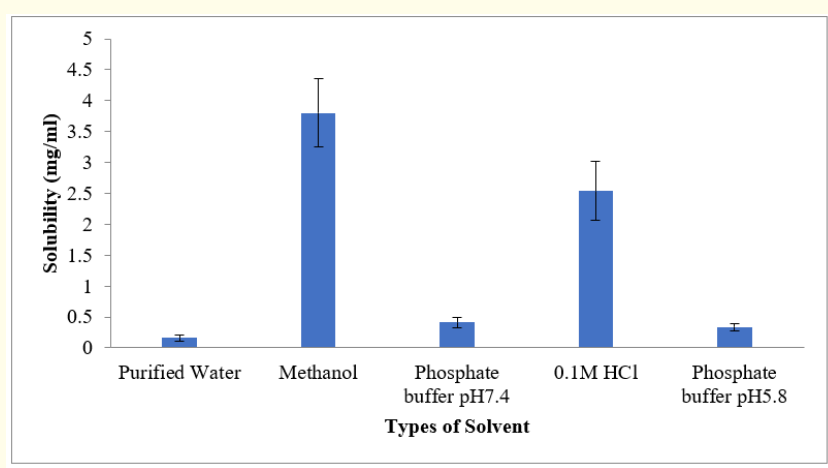

Figure 2: Solubility data of lamotrigine.

\begin{tabular}{|l|c|c|c|}
\hline \multirow{2}{*}{ Solvent } & \multirow{2}{*}{$\begin{array}{c}\text { Solubility } \\
\text { (mg/ml) }\end{array}$} & \multirow{2}{*}{ Report } & IP Reference \\
\cline { 3 - 4 } & & (Part of solvent \\
\cline { 3 - 4 } & required for 1 \\
\hline Purified Water & 0.165 & $\begin{array}{c}\text { Sparingly } \\
\text { soluble }\end{array}$ & 30 to 100 \\
\hline Methanol & 3.8 & Very soluble & 1 to 10 \\
\hline Phosphate \\
buffer, pH 7.4
\end{tabular}

Table 1: Solubility data of lamotrigine.

The solubility study of lamotrigine was performed in different solvents like purified water, methanol, phosphate buffer pH7.4, $0.1 \mathrm{M} \mathrm{HCl}$, phosphate buffer pH5.8 [4-6].

Preparation of calibration curve of lamotrigine in different solvent medium

Preparation of calibration curve of lamotrigine in methanol

For lamotrigine, calibration curve of lamotrigine was prepared in different solvent systems like methanol, phosphate buffer pH7.4. The different dilutions 5 to $30 \mu_{\mathrm{g}} / \mathrm{ml}$ were scanned and the calibration curve of lamotrigine was obtained. The $\lambda$ max was found to be $307 \mathrm{~nm}$ (Figure 3). This matched with official standard. In the case of lamotrigine the $\mathrm{r} 2$ value was found to be 0.9967 in the case of methanol and 0.9994 in the case of phosphate buffer $\mathrm{pH} 7.4$ solution [4,6] (Figure 4 and 5).

Preparation of Calibration curve of lamotrigine in Phosphate buffer pH 7.4

In the same way the calibration curve in Phosphate buffer $\mathrm{pH}$ 7.4 was prepared. The obtained result has been shown in figure 5 . It confirmed that the results were found to be similar $[6,7]$.

Nanosizing of lamotrigine by modified solvent evaporation method

During the nanosizing of lamotrigine after each sonication cycle the sample was observed for transmittance that confirm that as 


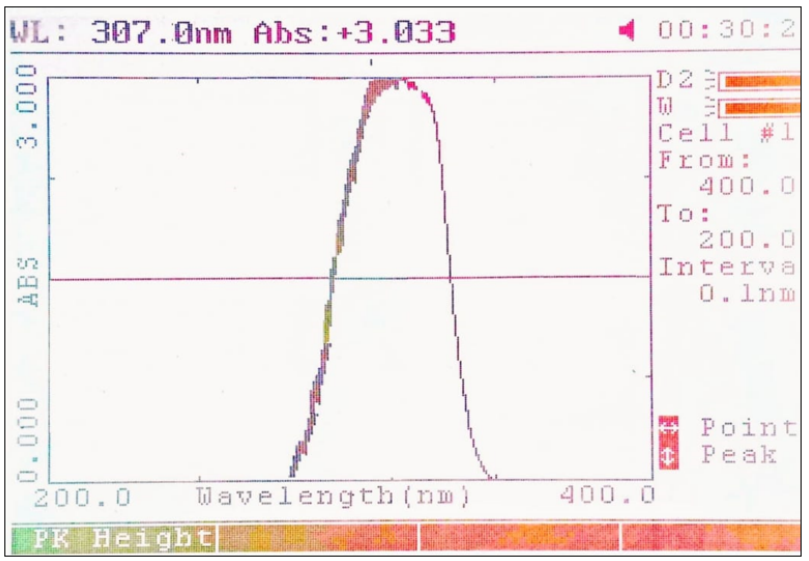

Figure 3: UV spectrum of lamotrigine in PBS, pH 7.4.

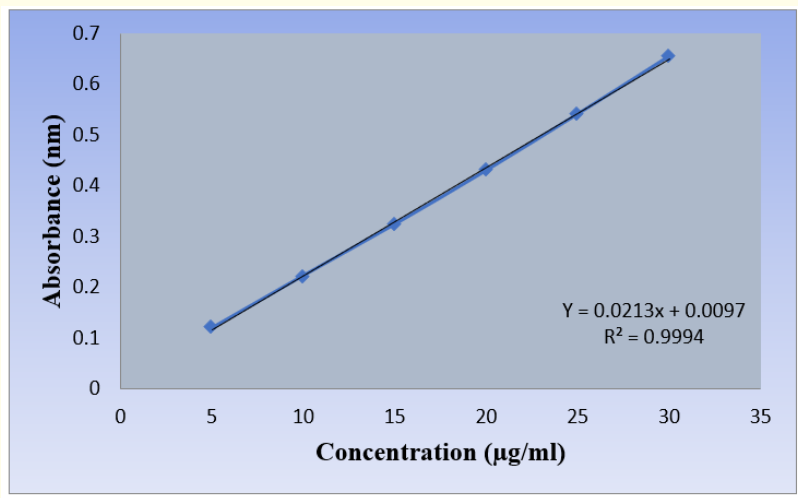

Figure 4: Calibration curve of lamotrigine in methanol.

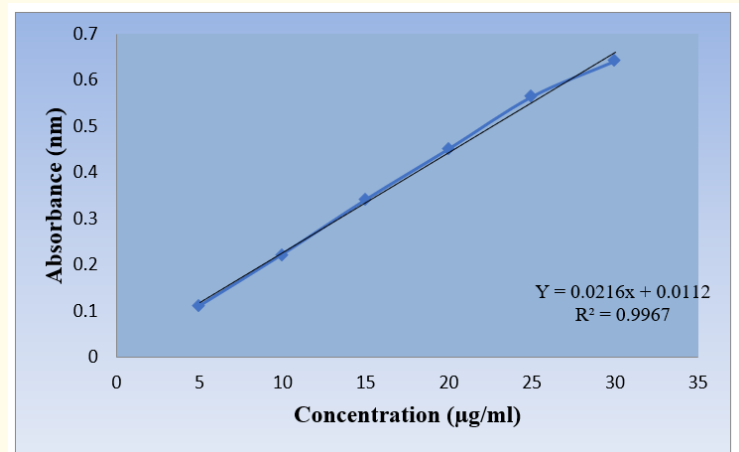

Figure 5: Calibration curve of lamotrigine in PBS pH 7.4. the number of sonication cycle increases the \% transmittance was increased. This was due to decrease in particle size and particles are now are in nanorange. Thus $\%$ transmittance shows the $\%$ of particles below $400 \mathrm{~nm}$ in bionanosuspension and \% blockade give an idea about the $\%$ of particles which are above $400 \mathrm{~nm}$. Thus the UV method has given an idea about particles in nano range. The nanosizing of drug particle was confirmed when the number of sonication cycles was increased up to 15 cycles as optimized, the $\%$ transmittance was found to increase proportionally as shown in figure 6 [9].

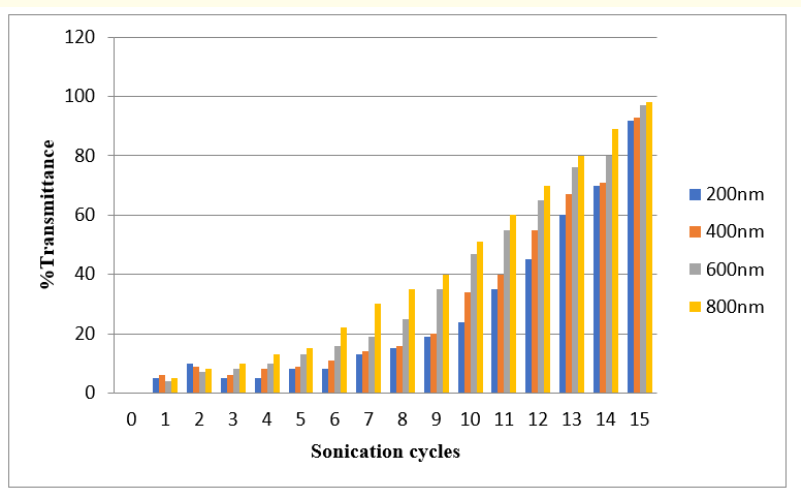

Figure 6: Nanosizing of lamotrigine and its charaterization by UV method.

\section{Physical characterization of nanosized model drug}

The recovered nanoparticles were observed for physical appearance.

\section{SEM characterization}

The nanosized drug was also characterized by SEM (scanning electron microscopy) testing [7]. The SEM image of the nanoparticles after nanosizing has showed that the particles with $100 \mathrm{~nm}$ and compared with SEM of drug particle before nanosizing. The SEM image of nanoparticles showed its crystalline nature (Figure $7 \mathrm{a}$ and $7 \mathrm{~b})$.

\section{Zeta particle size determination}

The nanosized drug was also characterized by Zeta sizer. The procedure of nanosizing has been summarized in flowchart 1 . The study was performed and reported (Varshney., et al. 2018). The 


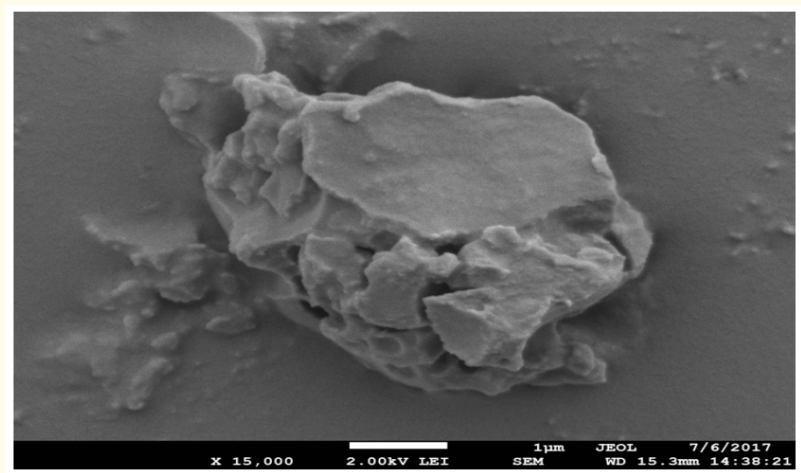

Figure 7: a. SEM of lamotrigine before nanosizing.

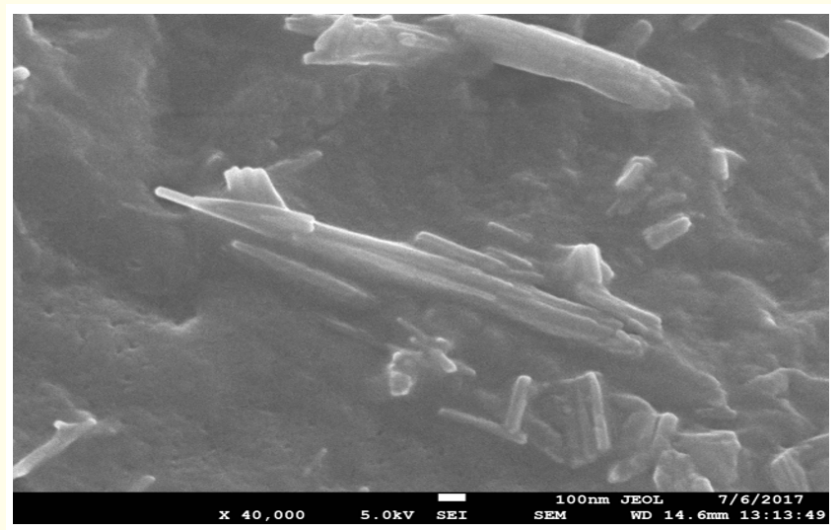

Figure 7: b. SEM of lamotrigine after nanosizing.

zeta particle size evaluation of model drug lamotrigine as well as nanosized lamotrigine revealed the drug particle size with 3109 $\mathrm{nm}(3.109 \mu \mathrm{m})$ was reduced to nano range with $119.7 \mathrm{Nm}$. From the obtained results it was concluded that the larger particles size of lamotrigine was reduced from micrometer to nanometer range because of the nanosizing during sonication $[9,10]$.

\section{In-vitro permeability study through the egg membrane}

In permeability study of lamotrigine a graph was plotted between the drug permeated at different time intervals through the egg shell membrane. The separated egg membrane was used as the permeation membrane which mimics the physiology of biomembrane. Cumulative graph showed an increase in Lamotrigine permeation with time. The permeation of drug also reveals that the particles are now in nano size range which can cross the biomembrane easily. Thus the nanosized drug are suitable for preparation of bionanosuspension as well as their passage through the biological membrane $[1,9,10]$ (Figure 8).

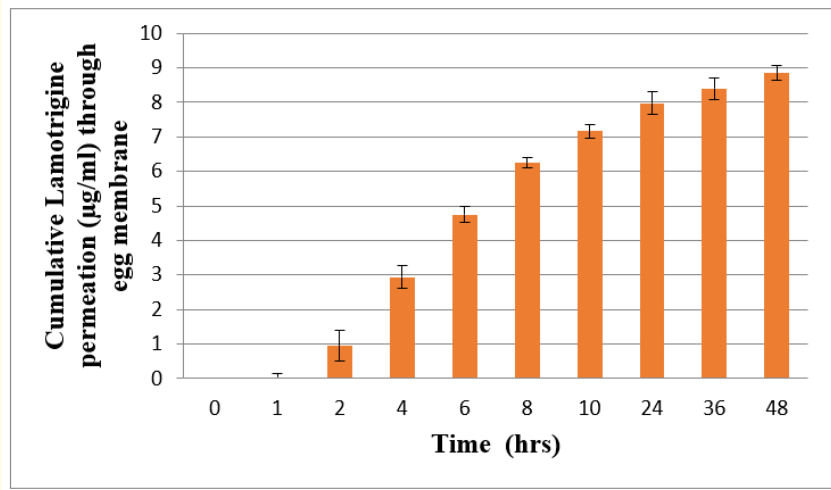

Figure 8: In-vitro permeation data of lamotrigine.

\section{Conclusion}

In this research the model drug was nanosized. The drug was physico-chemically characterized and showed the significant satisfactory results. The drug was characterized for SEM and zeta particle size which confirmed that the drug after nanosizing showed the satisfactory surface in SEM testing which also affected the solubility of the model drug. The larger particle sized before nanosizing in micrometer size range was easily converted into nanoparticles size range in nanometer. Thus the nano size range also improved the drug solubility as well as entrapment efficacy of the model drug. The nanosized drug was found to be significantly diffused through the egg biomembrane. This diffusion was in significant amount and confirmed that the nanosized drug easily pass through the biomembrane and can be targeted to desired sites in significant amount.

\section{Acknowledgement}

I wish to acknowledge Prof. Devender Pathak (Dean, Faculty of Pharmacy, UPUMS, Saifai), for encouraging me for the completion 
of research work. I want to also thank to SAIF, CDRI, Lucknow for providing me analytical testing facilities.

\section{Conflicts of Interest}

There was no any conflict of interest with this publication. There was no any financial support.

\section{Bibliography}

1. Madhav NVS., et al. "Formulation and evaluation of Duloxetine loaded bio-nano suspension for brain specificity via acoustic meatus". SOJ Pharmacy and Pharmaceutical Sciences 4.1 (2017): 1-5.

2. Madhav NVS., et al. "Development and evaluation of nanosized aripiprazole-loaded bioflexy films using a biopolymer from Lagenaria siceraria for brain delivery through orosoft palatal mucosal platform". Egypt Pharmaceutical Journal 16.1 (2017): 62-68.

3. Madhav NVS., et al. "A novel potent muco- bioadhesant polymer from seeds of Ricinus communis". World Journal of Pharmaceutical Sciences 3 (2014): 2154-2165.

4. Silverstein RM., et al. "Spectroscopic identification of organic compounds". 6th ed., New Delhi; Wiley India Pvt. Ltd (2010).

5. Sinko PJ. “Martin's, Physical pharmacy and pharmaceutical sciences". 5th Edition, B.I publications Pvt. Ltd, New Delhi (2006).

6. Richard A., et al. "Solid State Characterization of Pharmaceuticals". First Edition. Edited by Blackwell Publishing Ltd. Published by Blackwell Publishing Ltd. (2011).

7. Varshney S., et al. "Development and evaluation of bioflexy films using a novel biopolymer from Ananas cosmosus loaded with nanosized tiagabine". Egyptian Pharmaceutical Journal 17.1 (2018): 1-12.

8. Tyagi Y., et al. "Smart innovative approach for designing fluvoxamine loaded bio-nanosuspension for the management of depression". International Journal of Applied Pharmaceutics 11.1 (2019): 191-197.

9. Tyagi Y., et al. "Inbuilt novel bioretardant feature of biopolymer isolated from cucumis sativa for designing drug loaded bionanosuspension". Journal of Drug Assessment 9.1 (2020): 72-81.

10. Tyagi Y., et al. "Design of selegiline loaded bionanosuspension for the management of depression using novel bioretardant from Manilkara zapota". Drug Development and Industrial Pharmacy 45.8 (2019): 1351-1360.

11. Chatwal G., et al. "Instrumental methods of Chemical Analysis". 5th edition, Himalaya publishing house, New Delhi, 1.1-1.8 (2002): 2.566-2.570.

\section{Volume 5 Issue 7 July 2021 \\ (C) All rights are reserved by Sushant Kumar., et al.}

